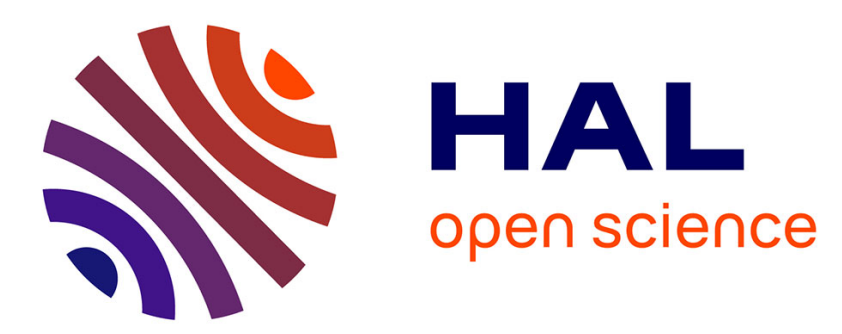

\title{
Minimizing the number of IGMP report messages for receiver-driven layered video multicasting
}

S. H. Shah Newaz, Youngin Bae, Jongmin Lee, Gyu Myoung Lee, Noel Crespi, Jun Kyun Choi

\section{- To cite this version:}

S. H. Shah Newaz, Youngin Bae, Jongmin Lee, Gyu Myoung Lee, Noel Crespi, et al.. Minimizing the number of IGMP report messages for receiver-driven layered video multicasting. VTC SPRING 2011: IEEE 73rd Vehicular Technology Conference, May 2011, Budapest, Hungary. pp.1-5, 10.1109/VETECS.2011.5956740 . hal-01304075

\section{HAL Id: hal-01304075 \\ https://hal.science/hal-01304075}

Submitted on 19 Apr 2016

HAL is a multi-disciplinary open access archive for the deposit and dissemination of scientific research documents, whether they are published or not. The documents may come from teaching and research institutions in France or abroad, or from public or private research centers.
L'archive ouverte pluridisciplinaire HAL, est destinée au dépôt et à la diffusion de documents scientifiques de niveau recherche, publiés ou non, émanant des établissements d'enseignement et de recherche français ou étrangers, des laboratoires publics ou privés. 


\title{
Minimizing the Number of IGMP Report Messages for Receiver-driven Layered Video Multicasting
}

\author{
S.H. Shah Newaz ${ }^{1 \& 2}$, Youngin Bae ${ }^{1}$, Jongmin Lee ${ }^{1}$, Gyu Myoung Lee ${ }^{2}$, Noël Crespi ${ }^{2}$, and Jun Kyun Choi ${ }^{1}$ \\ ${ }^{1}$ Dept. of Electrical Engineering, Korea Advanced Institute of Science and Technology (KAIST), Daejeon Rep. of Korea \\ ${ }^{2}$ Institut Telecom, Telecom SudParis, Evry, France \\ 1\&2newaz@kaist.ac.kr
}

\begin{abstract}
To manage multicast group in wired or wireless domain, the hosts need to send internet group management protocol (IGMP) report message when they are queried by the multicast routers. In fact, the bandwidth of wireless domain and mobile nodes' battery power are scarce resources; therefore, it can be big burden for the receiver-driven layered video multicasting (RLVM) receiving mobile hosts to follow the conventional report sending phenomenon, where a host needs to send a report message for subscribing each of the video layers. In this paper, we propose a mechanism for reducing the number of IGMP report messages for RLVM receiving hosts. The protocol overhead and efficiency are evaluated, and compared with conventional IGMPs. Our results show that applying proposed idea in IGMP the number of report messages can be minimized significantly for RLVM services.
\end{abstract}

Keywords- Multicast, Report suppression, RLVM, IGMP.

\section{INTRODUCTION}

In multicasting, the hosts which join in a group from directly attached network are queried by the multicast routers to learn whether at least one of the members of a specific group is receiving that multicast content or not. In response to the query, sent by multicast router, the hosts send report message. The role of this group management protocol is to maintain group membership for different sort of applications and service scenarios dynamically. However, it is a challenging task for the multicast router to keep tracking the presence of hosts for each multicast group when the number of multicast group or host increases rapidly [1].

In addition, another challenging issue in multicasting is heterogeneity of different display size of host terminals. Considering this heterogeneous display size issue in wired and wireless networks, receiver-driven layered multicasting (RLVM) technique has been introduced where the source transmits the video into several co-related and hierarchical layers [2]-[8]. According to this mechanism if a host only receives the first layer, the decoder of it can generate lowest quality signal. Whereas, if that host receives two layers, then the decoder of the host terminal can generate better signal by adding second layer information with the first layer. Therefore, for this innovative approach the multicast listener (host) has flexibility of choosing their compatible video based on their computational power, bandwidth (BW) and resource availability (e.g., buffer). In this approach, the source transmits different layers of the signal through multiple multicast groups and the receivers can receive different layers based on their requirement after joining that corresponding multicast groups [2]-[8]. Therefore, undeniably, the multicast router always needs to keep track the recipient hosts for each of the multicast groups of a particular video content, which has been scaled into different layers. For example, three separate layers can be generated from a video signal of MPEG-2 (I, P, B), or more precisely into five layers (I, P1, P2, $\mathrm{P} 3, \mathrm{E})[8]$. These five layers will be sent through five different multicast groups.

To state the significance of proposed idea, we would like to give an example. In general, let a layered video has $n$ layers. An $n$ layer video code can be modeled as follows [8]:

$$
\bar{L}=\left[L_{1}: L_{2}: \ldots . . L_{n}\right]
$$

Let's consider there are 10 hosts interested to watch a particular video content $X$, has $n$ layers. If each of the 10 hosts wants to receive all the layers, they have to join corresponding multicast group of each layer. Then according to the conventional rule of internet group management protocol (IGMP) version 1 (IGMPv1) and version 2 (IGMPv2), the multicast router should receive $n$ host membership reports after sending the general query, because IGMPv1 and IGMPv2 support report suppression [9], [10]. Whereas according to IGMP version 3 (IGMPv3) [11], the multicast routers should receive 10 host membership reports each of which composed of $n$ multicast group addresses. In case of IGMPv1 and IGMPv2 the protocol overhead is proportional to the number of multicast group that exists in a LAN [1]. In contrast, in case of IGMPv3 the protocol overhead is proportional to the number of hosts in a LAN subscribing in any multicast group. It is because the IGMPv3 does not support report suppression. For RLVM the number of IGMP report message will be increased, regardless of the attachment of host with wired or wireless networks. Our proposed idea of this paper can minimizes the number of IGMP report messages significantly; for example, based on the aforementioned scenario according to our proposed mechanism only 1 report message is required in IGMPv1 and IGMPv2, while in IGMPv3 the size of report message is reduced remarkably. Hence, protocol overhead is reduced significantly. In addition to that, our advocated mechanisms proposed for the RLVM in this paper is compatible with other existing congestion control algorithms designed for static layering (e.g. Receiver-driven Layered Congestion Control 
(RLC), Fair Layered Increase/ Decreased with Static Layering (FLID-SL)) [6].

Our proposed Number of Report Minimizing (NRM) scheme is tightly related with the concept of RLVM. It is needed to mention that the terms 'host' and 'multicast listener' have been used in this paper interchangeably. Evidently, when number of hosts or groups increases in conventional case, where each of the RLVM multicast groups is separately queried, the protocol overhead increases rapidly. To the best of our knowledge there is no specified recommendation is stated for group management of RLVM. Nevertheless, it has been mentioned clearly that each of the layer of RLVM content is transmitted through separate multicast group in [2][8].

The rest of the paper is organized as follows: the section II gives a brief description about the working principle of receiver-driven layered multicasting, IGMP version1, version 2 , and version 3. Section III summarizes the proposed idea, simulation is stated in section IV, and finally section V draws the conclusions.

\section{RELATED TECHNOLOGIES}

\section{A. Receiver-driven Layered Video Multicasting (RLVM)}

In this mechanism a raw video is encoded into several layers: a base layer (Layer 0 ) and 2 or 4 enhancement layers [8], [12]. The base layer, which provides basic quality of video, can be decoded independently, while the enhancement layers (i.e., Layer1,Layer 2 ), which are arranged in hierarchical fashion and give progressive refinement, cannot be decoded independently. To decode Layer 2 successfully, the receiver has to use Layer 0 and Layer 1 (see $\mathrm{L}_{0}, \mathrm{~L}_{1}$, and $\mathrm{L}_{2}$ in Fig.1). For experiencing better video quality, hosts need to subscribe not only base layer $\mathrm{L}_{0}$ but also enhancement layers such as $\mathrm{L}_{1}, \mathrm{~L}_{2}$, and $\mathrm{L}_{3}$ depending on their device's resolution, available network BW, and processing power. For example, if a host has large display size, it can periodically join to enhancement layer until it notices any packet loss. Evidently, when the receiver experiences any packet loss or congestion or insufficient buffer space, it can drop one or more enhancement layers from the top by leaving the corresponding multicast groups [2]-[8], [12].

\section{B. Query and Report Messages of IGMPs}

These three versions of IGMP, which has been specified for wired domain [13], have some similar features. For example, they have similar query and reply model. In a general Query message, the group address field is kept zero, while in the group-specific query the group address field contains group address of that group [10],[11]. In host membership report message, the group address field must contain the group address for which the host is sending the report. The multicast router sends the query messages to the multicast listeners (host) and the listeners send reply message in response to the query. IGMPv1 and IGMPv2 support report suppression mechanism, while the IGMPv3 does not. In IGMPv1, the interested multicast listener sends unsolicited membership message right after it joins in a particular

This work was supported by the IT R\&D program of MKE/KEIT. [KI001822, Research on Ubiquitous Mobility Management Methods for Higher Service Availability], S.Korea and the DiYSE (Do-it-Yourself Smart Experience), France. multicast group. This approach reduces join latency. Then again the IGMPv2, is the further improvement over IGMPv1, inherits the attributes of IGMPv1 and incorporates two other messages (i.e., General, and a Group-Specific Query) and two query intervals. However, the general query is same as the IGMPv1. One of the query intervals, which has longer interval, is used for general query, while the shorter query interval is used for Group-Specific Query.

1) IGMP V1: The router periodically sends General Group Queries to its attached subnet. When a host wants to join in a specific group, it sends IGMP report to the multicast router or it responds to the queries passively. Basically, in this version there are two mechanisms that help to avoid the implosion of concurrent reports when the host answers the group query. These are: delaying response and suppressing reports [9]. Delaying response means the host should response with delay rather than responding immediately. Report suppression means the host will stop sending the report message when it hears a report from other host joining the same group. In IGMPv1, the host does not send any report message when it leaves that group [9]. After a while the router assumes that the host is no longer listening from that group.

2) IGMP V2: Unlike IGMPv1, in IGMPv2 host sends leave group message when it decides to leave the group if it was the last member of that multicast group [10]. The report suppression mechanism is inherited from the IGMPv1 here. It is important to mention here that for IGMPv1 and IGMPv2 have 8 bytes fixed packet size [5].

3) IGMP V3: IGMPv3 inherits all the features of IGMPv2 but removes report suppression mechanism. In addition to that, it adds source filtering mechanism [11]. However, in the IGMPv3, the host sends a combined report of multiple groups within a single message [1], [11].

As the number of control message increases the protocol overhead also increases. Indeed, researchers are still thinking about the group management of IGMP in wireless domain. For instance, at present the IETF's Multicast Mobility (MULTIMOB) [13], an active working group, is considering the modification of IGMPv3/multicast listener discovery (MLD); so that these can suit well for the multicast group management in wireless domain.

\section{PROPOSED SCHEME FOR RLVM}

In RLVM, if any host wants to get the topmost layers $\left(L_{n}\right)$, obviously the host has to receive all the layers below $L_{n}$ for decoding $L_{n}$ successfully [2]-[8]. Therefore, based on this working principle of RLVM, for IGMPv1 or IGMPv2 a RLVM recipient host should send the report for only one multicast group which conveys topmost layer for that host at given time unless no similar report for that same multicast group or any report for other multicast groups, through which upper layers of same RLVM content is transmitted, is heard in proposed NRM. For IGMPv3, we propose that each RLVM recipient host should send the report for only one multicast group which conveys topmost layer for that host at a given time among all the received layers of a particular content $X$. 


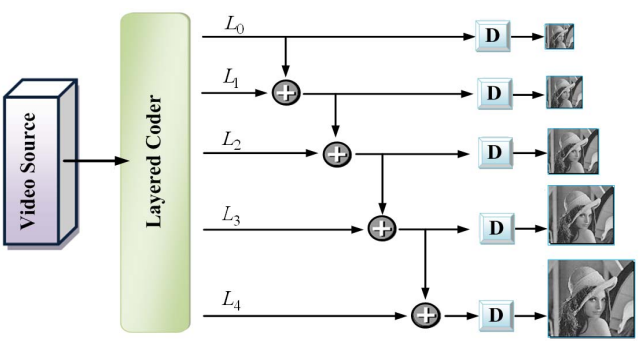

Figure 1. The layered video transission and recption scenario.

Hence at any given time the role of the multicast router, which may be installed inside the wireless base station or a separate node, is to find out the topmost layer having at least one host. We assume that at a given time a source produces 5 layers of content $X$ and transmits through 5 different multicasting addresses. Suppose at that time the set $M_{0}=\left\{q_{1}\right.$, $\left.q_{2}, \ldots, q_{m}\right\}$ is the set of total hosts who join in the multicast group of layer 0 ( $L_{0}$ or base layer ), and $M_{l}=\left\{q_{1}, q_{2}, \ldots, q_{n}\right\}$ be the set of total hosts who join in multicast group of layer 1 $\left(L_{1}\right)$. Similarly, the set $M_{2}=\left\{q_{1}, q_{2}, \ldots, q_{o}\right\}, M_{3}=\left\{q_{1}, q_{2}, \ldots, q_{p}\right\}$, and $M_{4}=\left\{q_{1}, q_{2}, \ldots, q_{r}\right\}$ be the set of total number of hosts who join in the multicast group of layer $2\left(L_{2}\right)$, layer $3\left(L_{3}\right)$, and layer $4\left(L_{4}\right)$ of that video content $X$ respectively. Where $m \geq n$ $\geq o \geq p \geq r$. Let's assume that $G_{0}, G_{1}, G_{2}, G_{3}$, and $G_{4}$ be the multicast group address for conveying $L_{0}, L_{1}, L_{2}, L_{3}$ and $L_{4}$ of that video content $X$ respectively. Then according to our proposed mechanism, the multicast router should expect report messages from the RLVM hosts based on the algorithm stated in Fig. 2. We further assume that this procedure can be triggered from the host terminals by sending the report for the topmost layer. On the other hand, technically, the multicast routers would understand this intention. It will abstain from sending any group specific query for the layers below the topmost layer as long as it receives the report messages for that layer. For example, at any given time if $L_{4}$ is the topmost layer having $r$ number of hosts, the set of total number of exempted hosts, $A$, of RLVM service for the content $X$ for IGMPv1 \&v2 is given by

$$
A=\left\{\bigcup_{a=0}^{4} M_{a}-q_{M_{4}, i}\right\}
$$

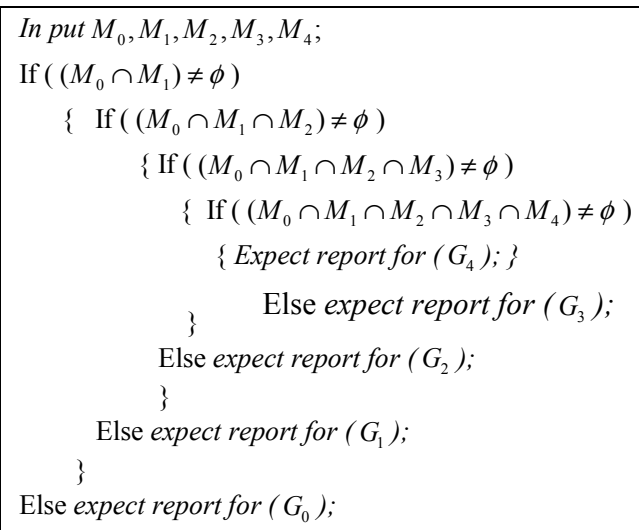

Figure 2. The process of choosing report expecting multicast group of any layered multicast video content $X$ at a multicast router.
Where $q_{M_{4}, i}$ is an entity of set $M_{4}$ and for $0<i \leq r$; Note that hosts of $G_{0}$ to $G_{3}$ can suppress the reports as long as a multicast router receives report for $G_{4}$. When there is no report from $G_{4}$ within that Max response time, the router should send group specific query for $G_{4}$ first. Again, if there is no report from $G_{4}$ recipient hosts within the Max response time [9]-[11], the router assumes that there is no host listening for $G_{4}$. In that case, the router should send group specific query for $G_{3}$ and starts expecting report for that group.

TABLE I
\begin{tabular}{|c|c|}
\hline Variable & NOTATIONS FOR ALGORITHM EXPLANATION \\
\hline$L_{\text {total }}$ & Total transmitted layers of content $X$ at a given time \\
\hline$t$ & Max response time \\
\hline$P_{G}^{X}$ & $\begin{array}{c}\text { Multicast group address used for conveying topmost layer of } \\
\text { a RLVM content } X \text { at a given time }\end{array}$ \\
\hline$P_{G p}^{X}$ & $\begin{array}{c}\text { Multicast group address used for conveying penultimate } \\
\text { topmost layer of a RLVM content } X \text { at a given time }\end{array}$ \\
\hline$G_{X}$ & $\begin{array}{c}\text { The set of multicast group addresses that are used for } \\
\text { conveying all the layers of content } X\end{array}$ \\
\hline$g_{X}$ & $\begin{array}{c}\text { The multicast group address of the topmost layer among all } \\
\text { the receiving layers of content } X \text { at host terminal at a given } \\
\text { time. }\end{array}$ \\
\hline$t_{1}$ & Last member query interval \\
\hline$a$ & A multicast packet receiving host \\
\hline
\end{tabular}

Fig. 3 states how these decisions can be made by multicast routers. In Fig. 4 (a) and (b) we present the algorithm of NRM scheme for IGMPv1\&v2 and IGMPv3 respectively. Using these algorithms host terminals can select a multicast group for which they have to send report message and can understand situation when they can suppress the reports for any multicast group.

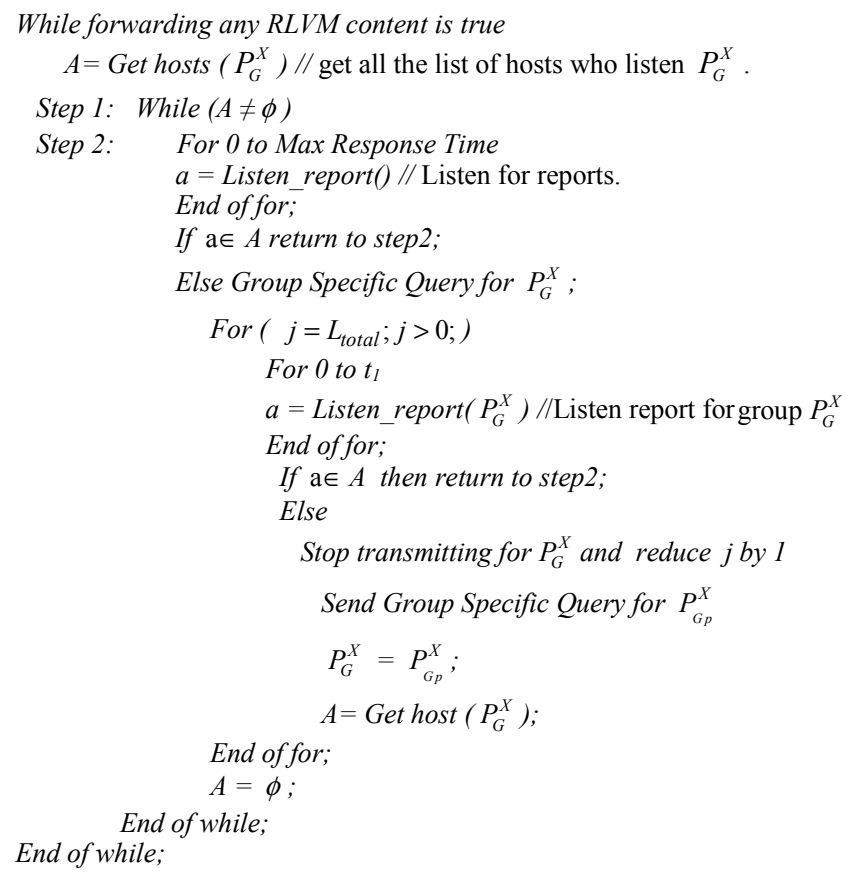

Figure 3. Algorithm for implemneting NRM scheme in multicast routers. 
While receiving any $R L V M$ content is true atIGMPv1or $v 2$ host terminal While receiving from $g_{X}$ is true

$S=$ random_time $f$ for $\left(g_{X}\right) / /$ Select random time for sending

report for $g_{X}$ within Max response time.

For 0 to $(S-\Delta t) \quad / / \Delta t$ is very small amount of time.

$G=$ Listen_report(); // Listen report of other hosts. End of for

If $G$ is equal $g_{X}$ or a group address conveys upper layer than

$g_{X}$ does, then stop sending report for $g_{X}$;

Else send report for $g_{X}$;

End of while;

End of while;

While receiving any $R L V M$ content is true

While receiving from $g_{X}$ is true

Wait until (Max response time $-\Delta t$ );

Put $g_{X}$ in report message; // A report message composed of

several multicast group addresses according to IGMPv3.

Send report within max response time,

End of while; // Receiving from $g_{X}$ can be false when host stop listen from that group.

End of while;

(b)

Figure 4. Algorithm for implemneting NRM scheme in host terminals, (a) NRM for IGMPv1\&v2, (b) NRM for IGMPv3.

\section{PERFormance EVAluation}

We compare the proposed NRM scheme with conventional query sending scheme for different versions of IGMP. To examine this, we conducted simulation with our developed $\mathrm{C}++$ simulator. First we analyze protocol overhead for proposed case and conventional case. We are also interested to understand efficiency, which can be defined as follows [5]:

$$
\text { Efficiency }=1-\left(\frac{\text { protocol overhead using NRM in IGMPv1\& } 2}{\text { protocol overhead in traditional IGMPv1\& } 2}\right) \text {. }
$$

TABLE II .NOTATIONS FOR NUMERICAL ANALYSIS

\begin{tabular}{|c|c|}
\hline Variable & Nomenclature \\
\hline$C$ & Number of host in a group (group size) \\
\hline$l$ & Number of layers of a RLVM content \\
\hline$N 1$ & RLVM contents' percentage in a multicast domain \\
\hline$(100-N 1)$ & $\begin{array}{c}\text { Independent video contents' Percentage in a multicast } \\
\text { domain }\end{array}$ \\
\hline
\end{tabular}

The (2) is also same for IGMPv3. Similar to [1], we assume that there is no loss of report or query messages and no communication delay between multicast router/base station and host. We consider that source can provide maximum 5 layers of multicast packets of a particular video content $X$. Initially, we further consider the number of interested hosts at all the layers of this content $X$ is equal. Considering the case without source filtering during a refresh period, approximate protocol overhead for IGMPv3 in that attached network can be calculated for conventional case as follows [1]:

$$
P_{I G M P V 3}=8 \text { bytes } \times C \times l \text {. }
$$

Whereas in the proposed case the protocol overhead of IGMPv3 can be calculated as follows:

$$
P_{I G M P V 3}^{*}=8 \text { bytes } \times C \text {. }
$$

Since the IGMPv1and IGMPv2 support report suppression, the protocol overhead is less in compare to IGMPv3. Then the protocol overhead for IGMPv1 and IGMPv2 approximately can be calculated for conventional case as follows [1]:

$$
P_{I G M P V 1 \& V 2}=8 b y t e s \times l \text {. }
$$

On the other hand, proposed case the protocol overhead for IGMPv1 and IGMPv2 can be calculated as follows:

$$
P_{I G M P V 1 \& V 2}=8 \text { bytes } \times 1 \text {. }
$$

The (6) shows that 8bytes has only multiplied by 1 . It is because the report suppression works in IGMPv1 and IGMPv2; therefore, only 1 report message will be sent to the multicast router among the topmost layers recipient hosts.

\section{A. Number of Layers of a RLVM Content}

We conducted the experiment to measure the relationship between the number of layers of the content $X$ and protocol overhead, keeping the group size same for all layers. Fig. 5 shows the relation between increment of layers of the content $X$ and protocol overhead. It states that when the number of layers of a video increases the protocol overhead increases in conventional IGMPv3, while using NRM in IGMPv3 the protocol overhead is invariant. In contrast, by applying NRM in IGMPv1\&v2, the protocol overhead also reduces. The Fig. 6 depicts the efficiency for both cases. Using (2) we compare the efficiency for proposed NRM over conventional mechanism. Interestingly, our obtained result shows similar efficiency of NRM over conventional IGMPv1\&v2 and IGMPv3.

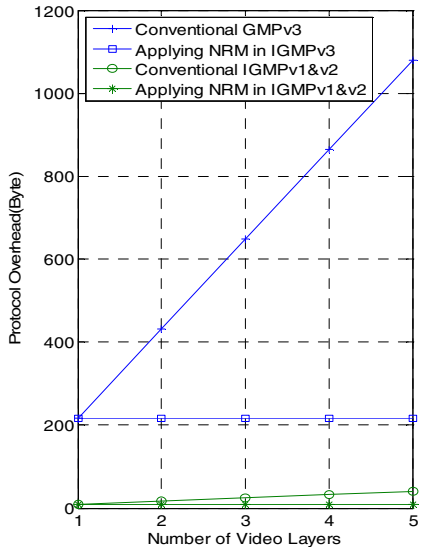

Figure 5: The relation between layer increment and protocol overhead.

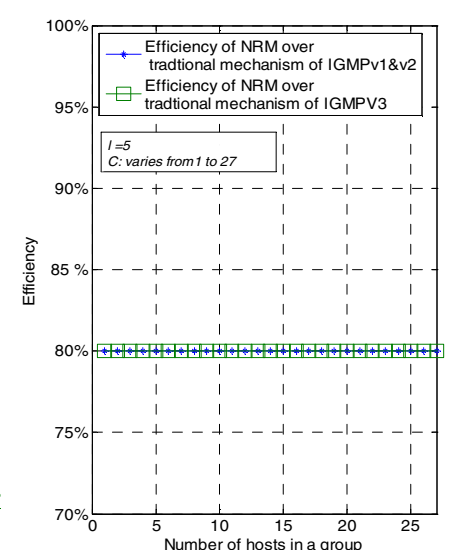

Figure 6: Efficiency of proposed NRM scheme over conventional mechanism.

\section{B. Group Size}

The Fig. 7 shows the relation between the number of hosts in topmost layer and protocol overhead in terms of proposed and conventional case for different versions of IGMP. We consider $l=5$ and $C$ varies from 0 to 27 . It can be observed from this figure that using NRM the protocol overhead can be reduced for IGMPv1\&v2. However; even if the number of host increases in topmost layer (number of host increases all the layers) for both conventional and proposed case, the protocol overhead remains constant. It is because IGMPv1 and IGMPv2 both support report suppression. We can also notice the conventional IGMPv3 performs worse. In contrast, proposed NRM performs well. 
Now we consider random joining and leaving scenario from each of the layers of content $X$. This experiment was conducted to understand at which situation the protocol overhead increases in conventional IGMPv3. We varied number of interested hosts for each layer randomly from 1 to 30. It can be observed from Fig. 8 when the number of participated hosts for topmost layers increases, protocol overhead increases rapidly in conventional IGMPv3. According to NRM, since only one multicast group address, which is the topmost layer conveying address for that host terminal at a given time, is put in the report message, the protocol over remain same no matter how many layers a host receives. During running this experiment, we also measured efficiency. The Fig.9 shows the efficiency of NRM over conventional IGMPv1\&v2, and IGMPv3. It indicates that as the number of host increases in topmost layer the efficiency of proposed NRM increases when applied in IGMPv3, while in IGMPv1\&v2 it is always invariant.

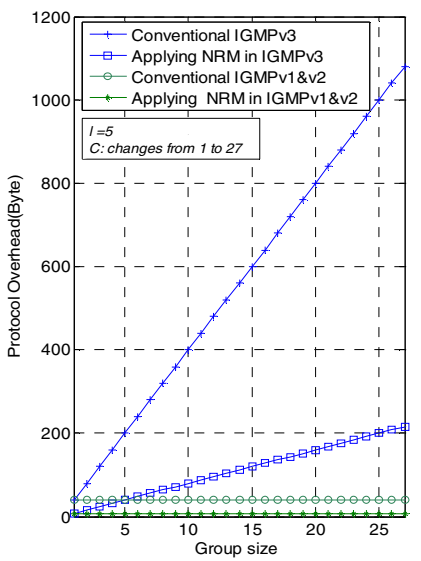

Figure 7: Group size vs. protocol overhead.

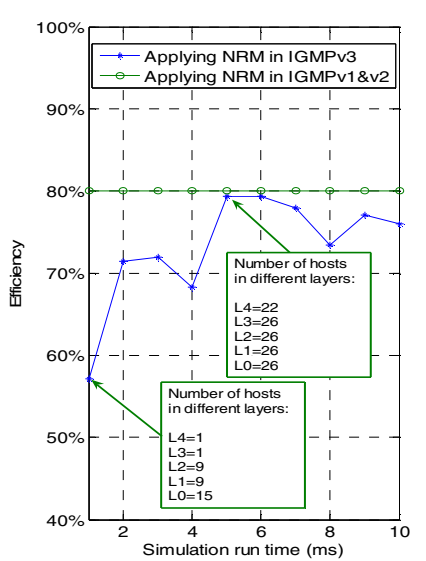

Figure 9: Impact of group size of each layer on efficiency.

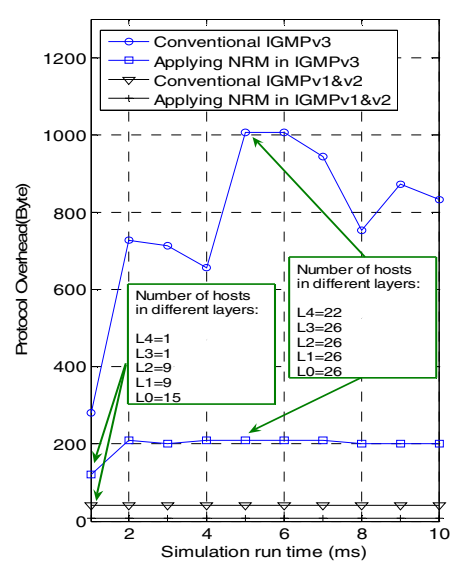

Figure 8: Impact of group size of each layer on protocol overhead.

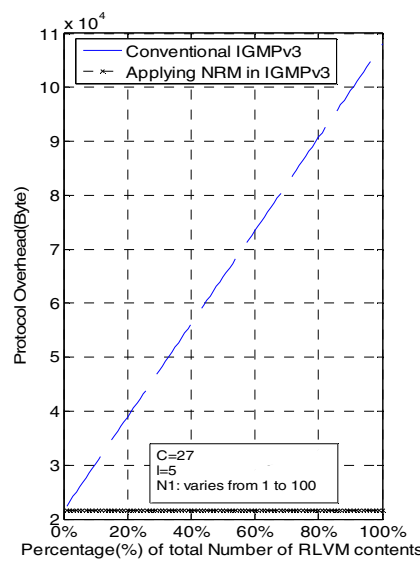

Figure 10: Percentage of RLVM content increment Vs protocol overhead in IGMPv3.
Inevitably, the higher the efficiency, the better the performance improvement of proposed NRM scheme over the group management schemes of conventional IGMPv3 and IGMPv1\&v2. For example, the $\mathrm{x} \%$ of efficiency means that using NRM in IGMPv3 the BW requirement is only $(100-\mathrm{x}) \%$ of conventional IGMPv3's. It indicates that the proposed NRM can increase BW utilizations.

\section{Percentage of RLVM Contents Forwarded by Multicast Routes}

Here we vary the percentage of RLVM content among all the multicast video contents forwarded by a multicast router at a given time. We assume on an average each multicast group has 20 hosts. The percentage of $N 1$ was varied from $1 \%$ to $100 \%$. From Fig. 10, it can be observed that protocol overhead increases significantly in conventional IGMPv3 as the percentage of RLVM content increases, whereas using NRM in IGMPv3 protocol overhead is invariant.

\section{CONCLUSIONS}

As RLVM can solve the heterogeneity problem of display size in multicast domains and ensures several flexibilities for the host terminals, this mechanism can be accepted widely. Therefore, whenever the demand for RLVM increases in any domain (wired or wireless), the multicast routers and hosts can adopt this proposed solution for reducing the number of report messages in that attached networks; so that, load on fixed or mobile hosts, and multicast routes can be reduced, and BW utilization can be improved.

\section{REFERENCES}

[1] Wanjiun Liao; De-Nian Yang; "Receiver-initiated group membership protocol (RGMP): a new group management protocol for IP multicasting", IEEE Trans. on Broadcasting, Vol. 50, Issue 3, Sep. 2004.

[2] Lucas, V.; Pansiot, J.-J.; Grad, D.; Hilt, B.; , "Fair Multicast Congestion Control (M2C)," INFOCOM Workshops 2009, IEEE , pp.1-6, 19-25 April 2009.

[3] Hua, K.A.; Tantaoui, M.A.; Tavanapong, W. "Video delivery technologies for large-scale deployment of multimedia applications"; Proceedings of the IEEE Volume 92, Issue 9, Sep 2004.

[4] Tran Ha NGUYEN, Kiyohide NAKAUCHI, MASATO KAWADA, Hiroyuki MORIKAWA, and Tomonori AOYAMA. "Rendezvous points based layered multicast". IEICE Trans.Commun Vol.E84-B, No.12 Dec. 2001.

[5] Steven McCanne, Martin Vetterli, Fellow, IEEE, and Van Jacobson, "Low-Complexity video coding for receiver-driven layered multicast" IEEE JOURNAL ON SELECTED AREAS IN COMMUNICATIONS, Vol. 15, No. 6, Aug. 1997.

[6] John W. Byers, Gavin Horn, Michael Luby, Michael Mitzenmacher, and William Shaverm, "FLID-DL: congestion control for layered multicast", IEEE JOURNAL ON SELECTED AREAS IN COMMUNICATIONS, Vol. 20, No. 8, Oct. 2002.

[7] Hon Sun Chiu; Yeung, K.L.; "Fast-response receiver-driven layered multicast", Computers and Communications, Proceedings. ISCC 2004. Ninth International Symposium on Vol. 2, No.28, pp.1032-1037, Jun. 2004.

[8] Tingting Zhang; Youshi Xu; "Unequal packet loss protection for layered video transmission", IEEE Transactions on Broadcasting, Vol. 45, Issue 2, pp.243-252, Jun. 1999.

[9] S. Deering, "Host extensions for IP multicasting," in IETF RFC 1112, Aug. 1989.

[10] W. Fenner, "Internet group management protocol, version 2," in IETF RFC 2236, Nov. 1997.

[11] B. Cain et.al B. "Internet group management protocol, version 3," IETF RFC 3376. Oct. 2002.

[12] Dapeng Wu; Hou, Y.T.; Ya-Qin Zhang; "Scalable video coding and transport over broadband wireless networks," Proceedings of the IEEE 802.16e std, Volume 89, Issue 1, Jan. 2001.

[13] http://tools.ietf.org/wg/multimob/charters. 